\title{
Nanotemplated lead telluride thin films
}

\author{
Xiaohong $\mathrm{Li}^{\mathrm{a}}$, Iris S. Nandhakumar ${ }^{\mathrm{a}, *}$, George S. Attard ${ }^{\mathrm{a}}$, Matthew L. Markham ${ }^{\mathrm{b}}$, \\ David C. Smith ${ }^{\mathrm{b}}$, Jeremy J. Baumberg ${ }^{\mathrm{b}}$
}

a School of Chemistry, University of Southampton, Southampton SO17 1BJ, UK

${ }^{\mathrm{b}}$ School of Physics and Astronomy, University of Southampton, Southampton SO17 1BJ, UK

\section{A R T I C L E I N F O}

\section{Article history:}

Received 14 November 2007

Received in revised form 9 September 2008

Accepted 10 September 2008

Available online 26 September 2008

\section{Keywords:}

Nanostructured materials

Templates

Electrodeposition

Thin films

IV-VI semiconductors

\begin{abstract}
A B S T R A C T
Direct lyotropic liquid crystalline templating has been successfully applied to produce nanostructured IV-VI semiconductor PbTe thin films by electrodeposition both on gold and n-type (100) silicon substrates. The PbTe films were characterized by transmission electron microscopy, X-ray diffraction and polarized optical microscopy and the results show that the films have a regular hexagonal nanoarchitecture with a high crystalline rock salt structure and exhibit strong birefringence. The optical absorption measurements using Fourier transform infrared spectroscopy show that the nanostructured PbTe films have a direct band gap of $0.37 \mathrm{eV}$.
\end{abstract}

(c) 2008 Elsevier Inc. All rights reserved.

\section{Introduction}

The increasing degree of miniaturization in components is presenting many new challenges to device engineering. One of these challenges is the ability to fabricate smaller structures down to nanometer length scales whose structure-property relationships are designed to yield devices with novel electronic, optical and magnetic properties that can impact on broad areas of technology. The chemist's approach to this challenge has been to develop synthetic strategies based on templating which rely on the self-assembly or self-organisation of nanometer-sized building blocks into organized connected arrays having long-ranged organization and order [1]. The rational design of functional materials is therefore an important goal that is becoming increasingly promise within nanotechnology. One templating strategy that offers considerable potential for nanofabrication is direct templating from lyotropic liquid crystalline (LC) phases. This enables the fabrication of threedimensional nanomaterials with well-defined and highly regular porous nanoarchitectures on the scale $2-20 \mathrm{~nm}[2,3]$. The method exploits the amphiphilic nature of non-ionic polyoxyethylene surfactants which can self-assemble into LC phases when mixed with water. By controlling the relative concentrations of the two components and temperature it is possible to produce condensed phases

\footnotetext{
* Corresponding author. Tel.: +44 2380 594484; fax: +44 2380593781 .

E-mail address: iris@soton.ac.uk (I.S. Nandhakumar).
}

with a wide range of different topologies which can be used as 'moulds' for nanocasting by either sol-gel reactions, or electrodeposition within the aqueous domain of the structure, resulting in materials with ordered architectures. Direct LC templating has been employed to produce a wide range of nanostructured materials [4-11].

In the present work we report the electrodeposition of high quality nanostructured thin films of lead telluride ( $\mathrm{PbTe}$ ) from the hexagonal $\left(\mathrm{H}_{\mathrm{I}}\right)$ LC phase of a non-ionic polyoxyethylene surfactant. PbTe is an attractive semiconductor material to cast with a porous nanoarchitecture because it offers the possibility of studying quantum confinement effects in this attractive semiconductor material and the potential to allow the nanoscale tunability of optical properties. Lead chalcogenides (IV-VI) are used extensively in infrared (IR) photodetectors [12], laser diodes [13] and thermophotovoltaic devices [14] due to their narrow band gaps and so a route to novel forms of these materials could impact on diverse areas of applications. Lead chalcogenides have been grown by various techniques including molecular beam epitaxy (MBE) [15], chemical vapour deposition [16], chemical bath deposition [17], electrodeposition [18] and more recently electrochemical atomic layer epitaxy (ECALE) [19]. In general, nanostructured lead chalcogenides are expected to show strong quantization effects by virtue of their large Bohr exciton radii (e.g. PbSe quantum dots have an exciton radius of $\sim 46 \mathrm{~nm}$ ) [20]. The comparatively small effective masses of electron and hole and the high dielectric constant in these materials would also enhance any quantum confinement behaviour observed. 


\section{Experimental}

\subsection{Materials}

Lead acetate $\left(\mathrm{Pb}\left(\mathrm{CH}_{3} \mathrm{COO}\right)_{2}\right.$, Aldrich 99.999\%), tellurium dioxide ( $\mathrm{TeO}_{2}$, Aldrich 99.999\%), nitric acid (Fisher 70\%), and non-ionic surfactant octaethyleneglycol monohexadecyl ether $\left(\mathrm{C}_{16} \mathrm{EO}_{8}\right.$, Nikko) were all used as received. All aqueous solutions were freshly prepared using reagent grade water $\left(15 \mathrm{M} \Omega \mathrm{cm}^{-1}\right.$ resistances) from an ELGA water purification system. All glassware was soaked overnight in a $3 \%$ Decon/deionised water solution, and washed thoroughly with deionised water prior to use.

\subsection{Liquid crystalline templating mixtures}

The LC templating mixtures used in the electrodeposition of nanostructured mesoporous PbTe films were obtained by mixing $50 \mathrm{wt} . \% \mathrm{C}_{16} \mathrm{EO}_{8}$ with $50 \mathrm{wt} . \%$ aqueous solution containing $30 \mathrm{mM}$ $\mathrm{Pb}\left(\mathrm{CH}_{3} \mathrm{COO}\right)_{2}$ and $1 \mathrm{mM} \mathrm{TeO}$ dissolved in $0.1 \mathrm{M} \mathrm{HNO}_{3}$. The mixture was sealed in a capped vial and allowed to stand in a thermostated oven at $60^{\circ} \mathrm{C}$ for $15 \mathrm{~min}$, which was followed by vigorous stirring with a glass rod. This heating/mixing process was repeated at least three times until a homogeneous mixture was obtained. Prior to the electrodeposition the mixture was heated to a temperature above the isotropic point and then cooled to room temperature at a rate of $0.2{ }^{\circ} \mathrm{C} \mathrm{min}^{-1}$ in order to improve the alignment of the LC mixture. The phase behaviour of LC templating mixtures was investigated with polarized optical microscopy (POM) using an Olympus BH-2 polarized light microscope equipped with a Linkam TMS90 heating stage and temperature control unit. The samples were placed between a glass slide and cover slip and viewed under POM by using heating/cooling rates of $0.2{ }^{\circ} \mathrm{C} \mathrm{min}^{-1}$ across the phase transition boundary.

\subsection{Electrodeposition}

Electrochemical depositions were performed using a three-electrode setup in combination with an Autolab potentiostat/galvanostat. A large area platinum gauze and saturated calomel electrode (SCE) were used as counter and reference electrodes respectively. Working electrodes were in the form of $200 \mathrm{~nm}$ thick gold films evaporated on microscope slides which were cleaned by sonication in isopropanol prior to use. The substrates were masked using polyimide tape to prevent edge effects during deposition, giving an exposed electrode area of $1 \times 1 \mathrm{~cm}^{2}$. The electrodeposition of $\mathrm{PbTe}$ films was carried out under potentiostatic and thermostatic control. Nanostructured PbTe films were deposited at potentials between $-0.40 \mathrm{~V}$ to $-0.48 \mathrm{~V}$ vs. SCE at $25^{\circ} \mathrm{C}$ from LC templating mixtures.

\subsection{Characterization}

The surface morphology and composition of the nanostructured PbTe films were characterized using a JSM-6500 F scanning electron microscope (SEM) equipped with energy dispersive X-ray (EDX) microanalysis (Oxford Inca 300) and operated at $15 \mathrm{kV}$. The nanostructure of the films was investigated using a JEOL 3010 transmission electron microscope (TEM) operating at an accelerating voltage of $300 \mathrm{kV}$. Wide-angle and low-angle X-ray diffraction (XRD) data were collected using a Siemens D5000 Xray diffractometer with $\mathrm{Cu} K \alpha$ radiation $(\lambda=1.5406 \AA$ ). The optical textures of the films were observed under POM with crossed polarizer. The polarizer before the sample is a fixed linear polarizer and the analyzer after the sample is a rotatable linear polarizer. The sample stage is also rotatable, perpendicular to the incident light.
Fourier transform infrared (FTIR) spectra were acquired on a Jasco 620 FTIR spectrometer. The PbTe films employed for FTIR measurements were deposited on double-side polished single crystalline ntype $\mathrm{Si}$ (100) wafers (phosphorus doped with resistivity of 1-10 $\Omega$ $\mathrm{cm}$, IDB Tech. Ltd). Before use, the wafer was degreased in isopropanol, cleaned in 2:1:8 $\mathrm{H}_{2} \mathrm{O}_{2}: \mathrm{HCl}: \mathrm{H}_{2} \mathrm{O}$ at $80^{\circ} \mathrm{C}$ for $15 \mathrm{~min}$, and then etched in $2 \mathrm{M}$ HF for $2 \mathrm{~min}$. Ohmic contacts to the Si substrates were made with Ga-In eutectic. The electrodeposition of PbTe films on $\mathrm{Si}$ substrates was carried out under the same deposit conditions and using the same LC templating mixtures.

\section{Results and discussion}

The electrochemical synthesis of stoichiometric films of nanostructured $\mathrm{PbTe}$ by direct LC templating required careful optimisation and control over a number of experimental parameters including deposition potential, choice of substrates, temperature, composition of LC templating mixtures, $\mathrm{pH}$, and concentration of precursor salts. As outlined in the experimental section stoichiometric hexagonal nanostructured $\mathrm{PbTe}\left(\mathrm{H}_{\mathrm{I}}-\mathrm{ePbTe}\right)$ thin films could be successfully deposited on gold substrates from LC template mixtures composed of $50 \mathrm{wt} . \% \mathrm{C}_{16} \mathrm{EO}_{8}$ and $50 \mathrm{wt} . \%$ aqueous solution containing $30 \mathrm{mM} \mathrm{Pb}\left(\mathrm{CH}_{3} \mathrm{COO}\right)_{2}$ and $1 \mathrm{mM} \mathrm{TeO}_{2}$ dissolved in $0.1 \mathrm{M} \mathrm{HNO}_{3}$.

To produce stoichiometric films of PbTe the composition of the deposited films was monitored as a function of the applied deposition potential and determined by EDX. As shown in Fig. 1 the optimum deposition potential was around $-0.43 \mathrm{~V}$ vs. SCE. This resulted in PbTe films with atomic compositions that were close to the stoichiometric ratio. PbTe films with a composition that was constant across the film were smooth and black in appearance. SEM images of the top view of such PbTe films (Fig. 2a) show a smooth and uniform texture compared to bulk PbTe films (Fig. 2b) which were produced from aqueous electrolyte without surfactant $\mathrm{C}_{16} \mathrm{EO}_{8}$. Fig. 3 shows a representative wide-angle XRD for $\mathrm{H}_{\mathrm{I}}-\mathrm{ePbTe}$ films deposited at $-0.43 \mathrm{~V}$ vs. SCE which indicates that the nanostructured film has a rock salt polycrystalline structure in accordance with other studies [18].

Low-angle XRD has been employed to examine the phase structure of the hexagonal LC mixtures and to determine the mesoporous nature of the nanotemplated PbTe films. Fig. 4 shows lowangle XRD diffractogram for the electrochemically deposited nanostructured $\mathrm{H}_{\mathrm{I}}-\mathrm{ePbTe}$ film and the LC template. This exhibits a single well-resolved first order diffraction peak $\mathrm{d}_{100}$ with a $d$-spacing of

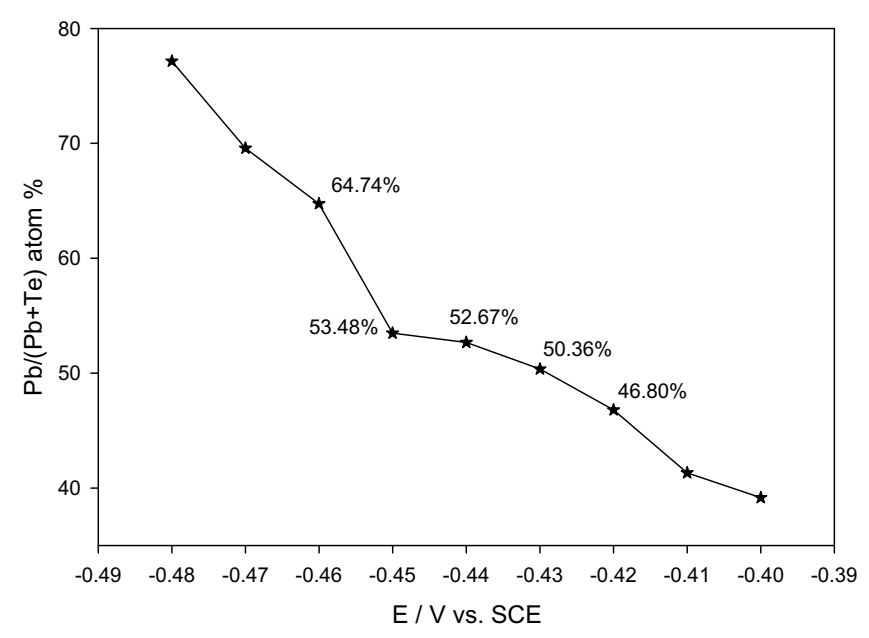

Fig. 1. Atomic percentages as a function of the applied potentials for electrodeposition of nanostructured PbTe films calculated from EDX analysis. 

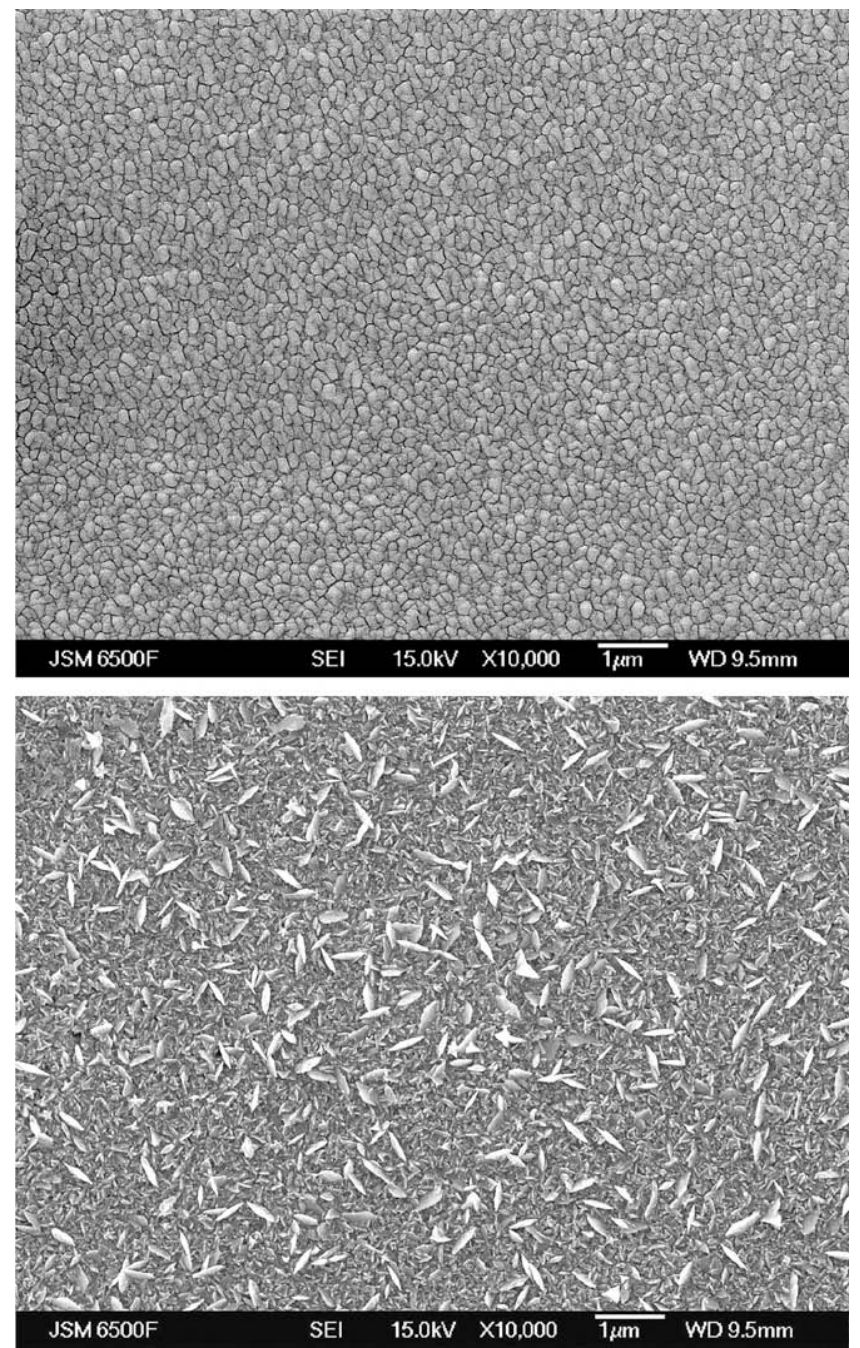

Fig. 2. SEM images of $\mathrm{H}_{\mathrm{I}}$-ePbTe films (a) deposited at $-0.43 \mathrm{~V}$ vs. SCE at $25^{\circ} \mathrm{C}$ from liquid crystalline templating mixtures and bulk films (b) deposited at $-0.43 \mathrm{~V}$ vs. SCE at $25^{\circ} \mathrm{C}$ from aqueous electrolyte in the absence of surfactant.

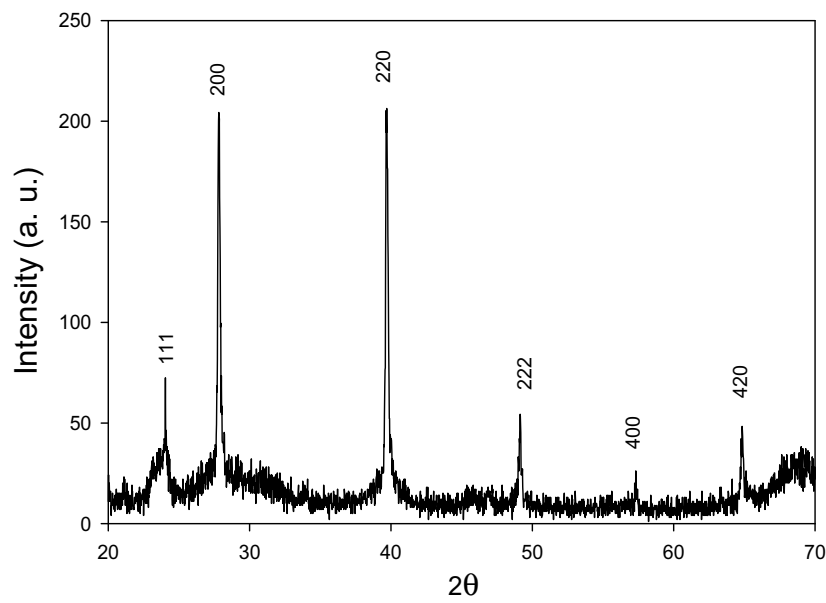

Fig. 3. Wide-angle XRD pattern for $\mathrm{H}_{\mathrm{I}}-\mathrm{ePbTe}$ films deposited at $-0.43 \mathrm{~V}$ vs. SCE at $25{ }^{\circ} \mathrm{C}$ from liquid crystalline templating mixtures.

$54 \pm 2 \AA$ that corresponds to a pore-to-pore distance of $62 \AA$. The fact that no primary reflection XRD peaks can be observed for reference PbTe films deposited in the absence of surfactant supports a

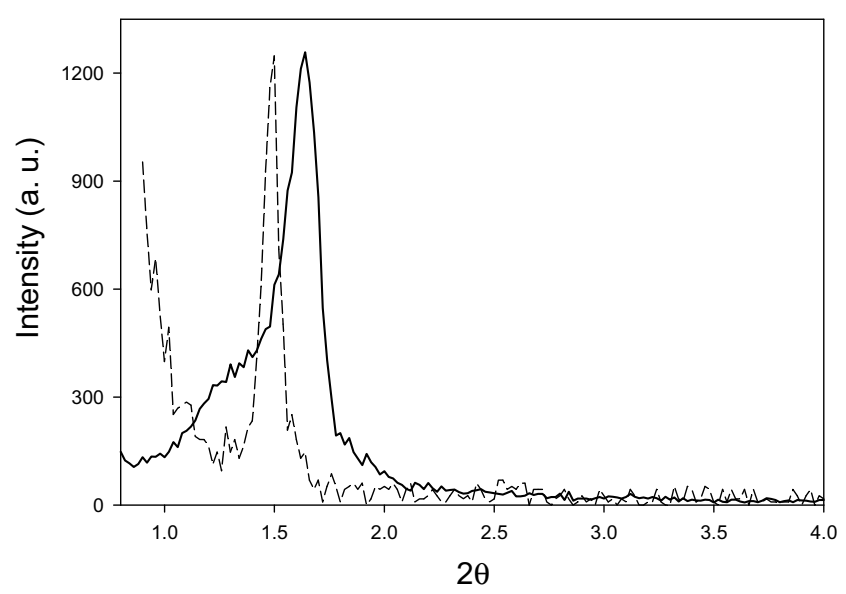

Fig. 4. Low-angle XRD pattern for $\mathrm{H}_{\mathrm{I}}$-ePbTe films (solid line) and liquid crystalline mixture (dash line) which was used to prepare the film.

direct LC templating process. The resulting semiconductor film exhibited the characteristic hexagonal symmetry and nanoscale dimensions of the LC template.

Direct evidence of the existence of a nanostructure as the result of the deposition of $\mathrm{H}_{\mathrm{I}}-\mathrm{ePbTe}$ was obtained by TEM. Fig. 5 shows micrographs of a $\mathrm{H}_{\mathrm{l}}$-ePbTe film electrodeposited at $-0.43 \mathrm{~V}$ vs. SCE. Fig. 5a shows clear nanoscale order with a periodic repeat distance of $58 \pm 2 \AA$. The hexagonal array of pores can be observed from the end-on view image in Fig. 5b. Analysis of the TEM images shows that the $\mathrm{H}_{\mathrm{r}}$-ePbTe film has an average pore diameter of $30 \pm 2 \AA$ and a pore-to-pore distance of $60 \pm 2 \AA$ which is close to the value obtained from the low-angle XRD.

In addition, when viewed through crossed polarizers the templated films exhibit strong form birefringence as would be expected for anisotropic nanostructures. The textures are similar to those of the LC templating mixtures with commonly fan-like or cross-like texture, which is a characteristic pattern of hexagonal phase $[21,22]$. Some large domains have been observed in the films as shown in Fig. 6. The appearance of such domains is due to the particular alignment of the pores in the films which indicates all the pores pointing in one particular direction with the same angle with respect to the substrates. As the sample is rotated the two adjacent domains go from light to dark and to light again with a $90^{\circ}$ period. It is clear from these observations that the birefringent nature of the hexagonal LC templates has been cast into PbTe films and the direct $\mathrm{LC}$ templating approach is capable of turning an optically isotropic material into an anisotropic metamaterial. Furthermore, since the dimension and topology of nanoarchitecture are under direct experimental control by the choice of surfactant and by the addition of co-solvents [2,6], this approach has advantage that it can be used to produce tunable birefringent materials for a specific applications such as phase matching devices [23].

Optical absorbance spectra of both bulk and nanostructured PbTe thin films were measured at room temperature using an FTIR spectrophotometer. The films were deposited on double-sided polished single crystalline n-type $\mathrm{Si}(100)$ wafers which are optically transparent to infrared radiation out to wavelengths beyond $14 \mu \mathrm{m}$ [24] and allow us to evaluate the absorption coefficient and band gap of the PbTe thin films. In order to ensure the nanostructure of the films on Si substrates the films were deposited under the same deposit conditions and using the same LC templating mixtures as those on gold substrates and also investigated by low-angle XRD. The appearance of a single diffraction peak $\mathrm{d}_{100}$ confirms the existence of the hexagonal nanostructure in these films. The thicknesses of bulk and $\mathrm{H}_{\mathrm{I}}$-ePbTe thin films were $597 \mathrm{~nm}$ and $1031 \mathrm{~nm}$ respectively as measured using SEM. Since $\mathrm{H}_{\mathrm{l}}-\mathrm{ePbTe}$ film 

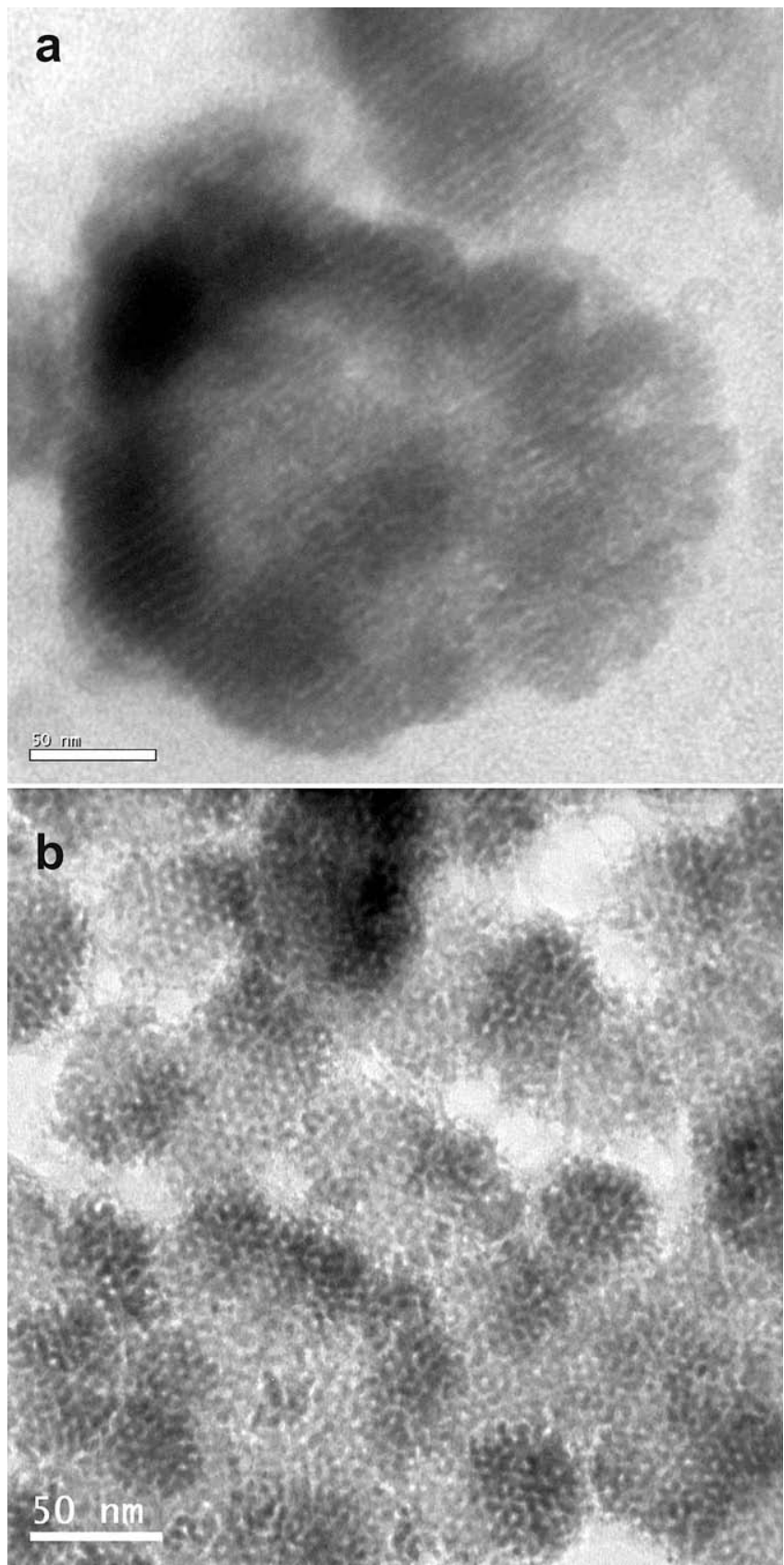

Fig. 5. TEM images of $\mathrm{H}_{\mathrm{I}}$-ePbTe films deposited at $-0.43 \mathrm{~V}$ vs. SCE at $25{ }^{\circ} \mathrm{C}$ from liquid crystalline templating mixtures: (a) side view of array of pores; (b) end-on view of pore structure.

with a perfect hexagonal nanostructure contains $77.3 \%$ (by volume) PbTe and 22.7\% (by volume) voids [5], the effective thickness of $\mathrm{H}_{\mathrm{I}}$-ePbTe film is estimated to be $797 \mathrm{~nm}$. Fig. 7 shows the variation of the absorption coefficient as a function of wavelength for bulk and $\mathrm{H}_{\mathrm{I}}$-ePbTe films deposited on Si substrates. It is observed that both films show high absorption coefficient and exhibit strong absorption at wavelength below $4 \mu \mathrm{m}$, which is near the room temperature band gap of PbTe [25]. Fig. 8 shows the plot of $(\alpha h v)^{2}$ versus photon energy, hv, for the electrodeposited bulk and $\mathrm{H}_{\mathrm{I}}$-ePbTe films. For both of the films, $(\alpha \mathrm{h} v)^{2}$ was a linear function of the photon energy, as would be expected for a direct band gap semiconductor [26]. The band gap value of the films can be obtained by linear extrapolation of the curve to zero absorption, giving the direct band gap $E_{\mathrm{g}}$ of $0.32 \mathrm{eV}$ and $0.37 \mathrm{eV}$ for bulk and $\mathrm{H}_{\mathrm{I}}-\mathrm{e}$ PbTe films respectively.
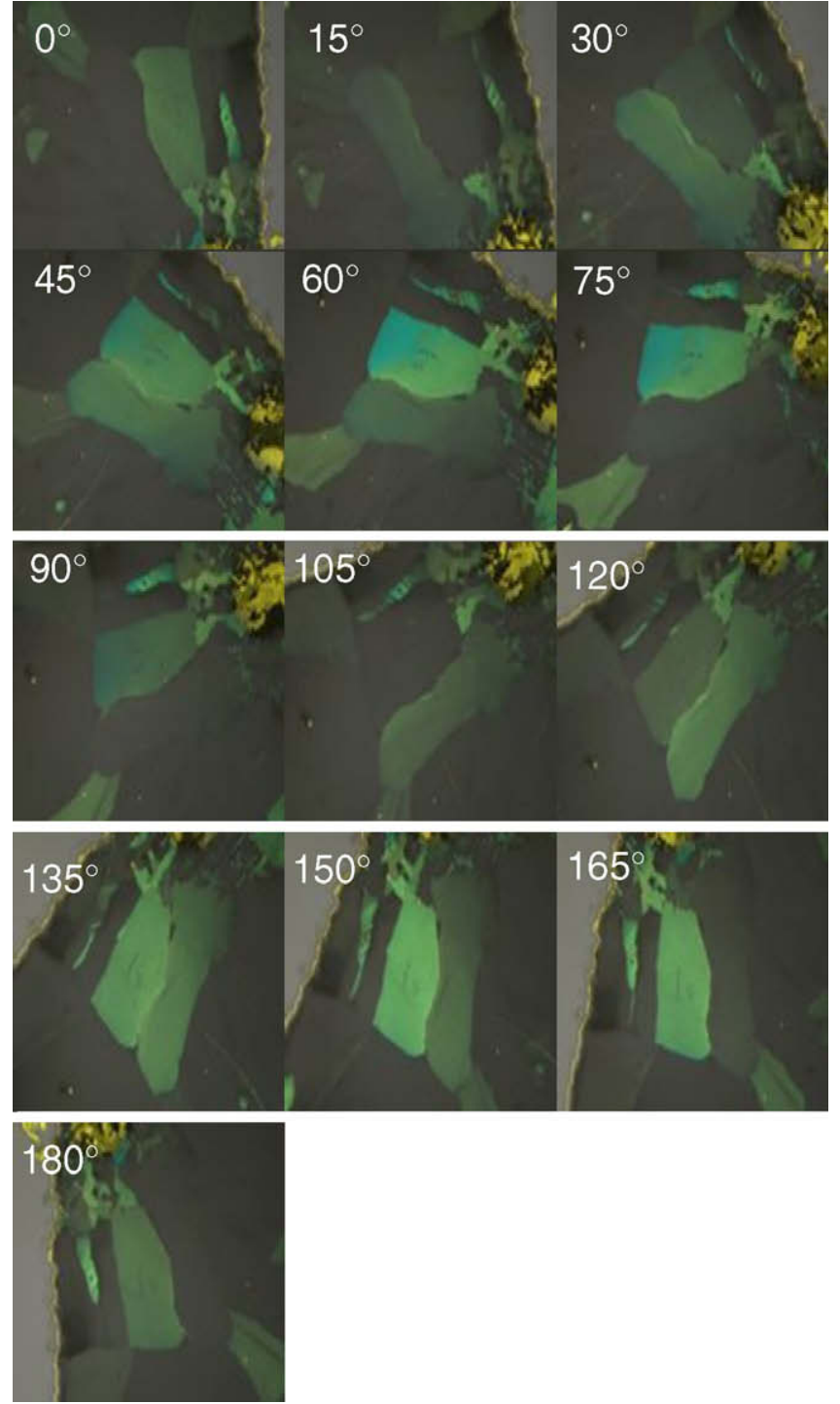

Fig. 6. Optical micrographs of two large adjacent birefringent domains of $\mathrm{H}_{\mathrm{I}}-\mathrm{ePbTe}$ film with cross polarized illumination and observation with a rotation of the sample between $0^{\circ}$ and $180^{\circ}$ with $15^{\circ}$ steps. Image scale is $1 \mathrm{~mm}$.

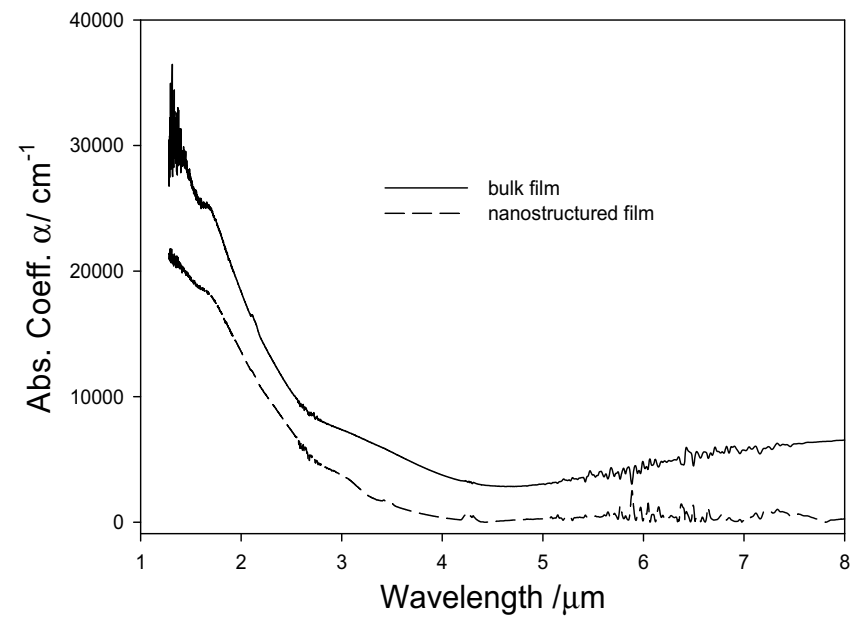

Fig. 7. Absorption coefficient $(\alpha)$ versus wavelength plots for bulk (solid line) and nanostructured (dashed line) PbTe films deposited at $-0.43 \mathrm{~V}$ vs. SCE at $25{ }^{\circ} \mathrm{C}$ on nSi (100) substrate. 


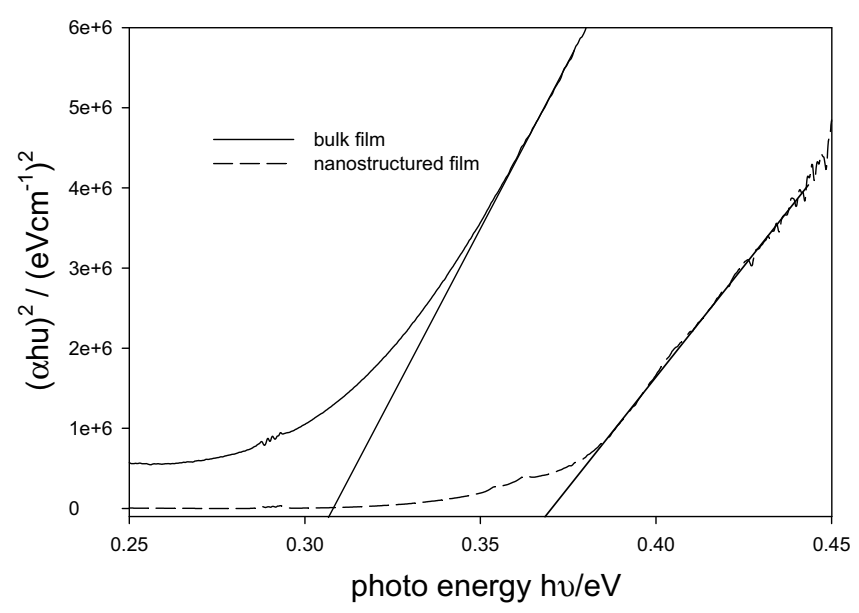

Fig. 8. $(\alpha h v)^{2}$ versus photon energy, hv, plots for determination of band gap of bulk (solid line) and nanostructed (dashed line) PbTe films deposited at $-0.43 \mathrm{~V}$ vs. SCE at $25^{\circ} \mathrm{C}$ on $\mathrm{n}-\mathrm{Si}(100)$ substrate.

\section{Conclusions}

We have reported the production of high quality thin films of nanostructured $\mathrm{PbTe}$ by electrodeposition from the hexagonal liquid crystal phase of $\mathrm{C}_{16} \mathrm{EO}_{8}$, a non-ionic polyoxyethylene surfactant which acts as a template for the formation of the nanostructure. The material produced was found to be stoichiometric with a composition of 50.36 at.\% $\mathrm{Pb}$ and 49.64 at.\% Te. The results of TEM, POM and XRD have shown that the films have a hexagonal nanostructure and high crystalline rock salt structure. The optical measurements show that the obtained nanostructured PbTe films are highly birefringent and of good quality with a direct band gap value of $0.37 \mathrm{eV}$ that is blue shifted in comparison to bulk films, $0.32 \mathrm{eV}$.

\section{Acknowledgment}

We are grateful for the funding from the EPSRC (grant code GR/ S02662).

\section{References}

[1] J.J. Baumberg, Nat. Mater. 5 (2006) 2.

[2] G.S. Attard, J.C. Glyde, C.G. Göltner, Nature 378 (1995) 366.

[3] G.S. Attard, P.N. Bartlett, N.R.B. Coleman, J.M. Elliott, J.R. Owen, J.H. Wang, Science 278 (1997) 838.

[4] G.S. Attard, C.G. Göltner, J, M. Corker, S. Henke, R.H. Templer, Angew, Chem. Int. Ed. Engl. 36 (1997) 1315.

[5] J.M. Elliott, G.S. Attard, P.N. Bartlett, N.R.B. Coleman, D.A.S. Merckel, J.R. Owen, Chem. Mater. 11 (1999) 3602.

[6] P.N. Bartlett, P.N. Birkin, M.A. Ghanem, P. de Groot, M. Sawicki, J. Electrochem. Soc. 148 (2001) C119.

[7] I.S. Nandhakumar, J.M. Elliott, G.S. Attard, Chem. Mater. 13 (2001) 3840.

[8] T. Gabriel, I.S. Nandhakumar, G.S. Attard, Electrochem.Commun. 4 (2002) 610.

[9] P.N. Bartlett, B. Gollas, S. Guerin, J. Marwan, Phys. Chem. Chem. Phys. 4 (2002) 3835.

[10] L. Huang, H. Wang, Z. Wang, A. Mitra, K.N. Bozhilov, Y. Yan, Adv. Mater. 14 (2002) 61 .

[11] H. Luo, J. Zhang, Y. Yan, Chem. Mater. 15 (2003) 3769.

[12] T.H. Johnson, Int. Soc. Opt. Eng. 443 (1984) 60.

[13] B. Spranger, U. Schiessel, A. Lambrecht, H. Böttner, M. Tacke, Appl. Phys. Lett. 53 (1988) 2582.

[14] H. Zogg, S. Bluner, C. Maissen, J. Mazek, A.N. Tiwari, IEEE Trans. Electron Dev. 38 (1991) 1110.

[15] H. Zogg, C. Maissen, J. Mazek, T. Hoshino, S. Bluner, A.N. Tiwari, Semicond. Sci. Technol. 6 (1991) C36.

[16] N.I. Fainer, M.L. Kosinova, Y.M. Rumyantsev, E.G. Salman, F.A. Kuznetsov, Thin Solid Films 280 (1996) 16.

[17] S. Gorer, A. Albu-Yaron, G. Hodes, Chem. Mater. 7 (1995) 1243.

[18] H. Saloniemi, T. Kanniainen, M. Ritala, M. Leskelä, Thin Solid Films 326 (1998) 78.

[19] R. Vaidyanathan, S.M. Cox, U. Happek, D. Danga, M.K. Mathe, J.L. Stickney, Langmuir 22 (2006) 10590.

[20] F.W. Wise, Acc. Chem. Res. 33 (2000) 773.

[21] S.T. Hyde, in: K. Holmberg (Ed.), Handbook of Applied Surface and Colloid Chemistry, John Wiley \& Sons, Chichester, 2002. pp. 299-332.

[22] Ö. Dag, S. Alayoğlu, C. Tura, Ö. Celik, Chem. Mater. 15 (2003) 2711.

[23] L.A. Golovan, V.Yu. Timoshenko, A.B. Fedotov, L.P. Kuznetsova, D.A. SidorovBiryukov, P.K. Kashkarov, A.M. Zheltikov, D. Kovalev, N. Künzner, E. Gross, J. Diener, G. Polisski, F. Koch, Appl. Phys. B 73 (2001) 31.

[24] H. Gómez, R. Henríquez, R. Schrebler, R. Córdova, D. Ramírez, G. Riveros, E.A. Dalchiele, Electrochim. Acta 50 (2005) 1299.

[25] J.E. Murphy, M.C. Beard, A.G. Norman, S.P. Ahrenkiel, J.C. Johnson, P. Yu, O.I. Mićić, R.J. Ellingson, A.J. Nozik, J. Am. Chem. Soc. 128 (2006) 3241.

[26] J.I. Pankove, Optical Processes in Semiconductors, Prentice-Hall, Englewood Cliffs, 1971. pp. 34-81. 